\title{
A large outbreak of conjunctivitis caused by a single genotype of Neisseria gonorrhoeae distinct from those causing genital tract infections
}

\author{
D. B. $\mathrm{MAK}^{1 *}$, D. W. SMITH ${ }^{2}$, G. B. HARNETT ${ }^{2}$ AND A. J. PLANT 3 \\ ${ }^{1}$ Kimberley Public Health Unit, West Kimberley House, Loch St, Derby, Western Australia 6728, Australia \\ ${ }^{2}$ Division of Microbiology and Infectious Diseases, The Western Australian Centre for Pathology and \\ Medical Research \\ ${ }^{3}$ Division of Health Sciences, Curtin University of Technology, Bentley, WA 6845
}

(Accepted 7 January 2001)

\section{SUMMARY}

Several epidemics of gonococcal conjunctivitis have occurred in Aboriginal populations in Central Australia. In 1997, the first outbreak in the Kimberley region of Western Australia occurred, spreading to Central Australia with a total of 447 cases. A genotyping method was applied directly to DNA extracted from patient samples to characterize the gonococcus causing the epidemic and to compare it with contemporaneous genital isolates. Those positive conjunctival specimens from Kimberley and Central Australia that could be genotyped were all indistinguishable, but were distinct from the genital gonococci, even when they shared the same auxotype and serotype. This suggested that the outbreak was due to a single genotype of Neisseria gonorrhoeae that had probably been carried between communities by infected individuals. We did not find evidence to support the existence of a genital reservoir of the types causing epidemic gonococcal conjunctivitis.

\section{INTRODUCTION}

Gonococcal conjunctivitis is a highly infective, sightthreatening infection. It is uncommon in developed countries but has caused large outbreaks of disease in the developing world. In Australia, several outbreaks have been described in remote Aboriginal communities in Central Australia [1-4]. The largest of these occurred in 1991 with 432 cases [3] and in 1997 with 447 cases [4]. The 1997 epidemic began with a cluster 105 clinical cases in the Kimberley region of Western Australia, nearly all of which occurred between midMarch and mid-April. This was followed by a larger outbreak in Central Australia beginning in mid-April and continuing to the end of June, with a combined total of 447 cases in Kimberley and Central Australia.

Limited auxotyping and serotyping were carried out and all conjunctival isolates were of the Wt/IB3

* Author for correspondence. auxotype/serovar. This auxotype/serovar was also the predominant one in a small number of genital gonococcal isolates, suggesting a possible genital reservoir for the conjunctival gonococci [4]. The occurrence of outbreaks of conjunctivitis in populations in Australia [1-5] and Africa [6] that have high rates of genital gonorrhoea also support this possible association. However, outbreaks of gonococcal conjunctivitis are not preceded by increases in genital gonorrhoea and their seasonality and transmission patterns are quite distinct from those of genital infections $[3,4,6]$. The Kimberley region of Western Australia has the highest reported rates of gonorrhoea in Australia [5], but no gonococcal conjunctivitis outbreaks had been reported prior to 1997.

We undertook a more detailed study of 46 laboratory-confirmed Kimberley cases. Isolates were not available from the majority of the cases, which had been diagnosed by polymerase chain reaction 
(PCR) amplification and detection of gonococcal DNA. A genotyping scheme for genital gonococcal infections has been described [7] and was modified for use on conjunctival samples. We were able to compare the genotypes found in conjunctival samples with those in contemporaneous genital isolates and sporadic conjunctivitis cases, as well as with eye and genital isolates from Central Australia.

\section{METHODS}

The Kimberley outbreak occurred largely in four communities in the southeast of the region. Cases consisted of all patients from these communities in whom a clinical diagnosis of gonococcal conjunctivitis was made between March 1998 and September 1998 that were confirmed by culture of Neisseria gonorrhoeae or by detection of gonococcal DNA following PCR amplification. Patients from neighbouring communities who were known to have had direct contact with cases from these communities, and in whom a clinical diagnosis of gonococcal conjunctivitis was confirmed by detection of Neisseria gonorrhoeae using PCR or culture during the above timeframe, were also considered as cases.

Information about cases was collected prospectively during the outbreak from local health services staff and from notifications received from laboratory and medical practitioners.

Community population data were collected by local census during June 1997, using health service and community administration records. Due to mobility between communities, variable English literacy rates and known problems with national census data in these communities, this was thought to be more accurate than Australian Bureau of Statistics census data.

Conjunctival swabs were taken for PCR using a cotton-tipped wire swab, which was returned to a dry container for transport to the laboratory at $4{ }^{\circ} \mathrm{C}$. Diagnostic testing by PCR was performed at the PathCentre laboratory in Perth. Samples were centrifuged at $14000 \mathrm{rpm}$ for $20 \mathrm{~min}$ and the pellets extracted using Chelex 100 resin (Bio-Rad Laboratories, Hercules, CA, USA) [8]. An in-house nested PCR was performed using primers directed at the cryptic plasmid of $N$. gonorrhoeae [9]. All positive samples were retested using two other nested PCRs directed at two separate target sequences within a $N$. gonorrhoeae specific probe [10]. Samples were reported positive only if they were positive with all of the PCRs.

Swabs for microscopy and culture were also taken if specimens could be transported to a laboratory within $48 \mathrm{~h}$. A conventional cotton-tipped swab was used, transported in Ames (charcoal-containing) transport medium at room temperature, and cultured at the nearest regional PathCentre laboratory using standard methods. However specimens from these remote communities did not usually have gonococcal culture performed because unavoidable delays in transporting samples to the regional laboratory meant they were unsuitable.

Conventional typing of gonococci by serotyping and auxotyping, could not be undertaken as the majority of cases either did not have a culture performed or were culture negative. Most of the isolates that had been obtained had been discarded. However, DNA extracts from the majority of the original samples were still available. To further investigate the outbreak, a published gonorrhoea genotyping technique [7] was modified to enable Kimberley gonococci to be detected and typed. Extracted DNA was amplified using a double PCR directed at an $841 \mathrm{bp}$ region of the porin gene, using primers designed in-house:

Primer A Gono 158: 5'-GGC GTA CAA ACT TAC CGT TCT GT-3'

Primer B Gono 981 : 5'-CCG CAC CGA CAA CCA CTT-3'

Amplification reactions contained $1 \mathrm{U}$ of TaqGold in $1 \times$ buffer (PE Applied Biosystems, Foster City, CA, USA) with $2.5 \mathrm{~mm} \mathrm{MgCl}, 0.2 \mathrm{~mm}$ dNTPs and $0.2 \mu \mathrm{M}$ of primers in a $20 \mu \mathrm{l}$ volume. An initial cycle of $15 \mathrm{~min}$ at $94{ }^{\circ} \mathrm{C}$ was followed by 45 cycles of $30 \mathrm{~s}$ at $94{ }^{\circ} \mathrm{C}$, $30 \mathrm{~s}$ at $55^{\circ} \mathrm{C}$ and $60 \mathrm{~s}$ at $72{ }^{\circ} \mathrm{C}$, and then a final cycle of $7 \mathrm{~min}$ at $72^{\circ} \mathrm{C}$. A $0 \cdot 4 \mu 1$ aliquot was then transferred to a new mix tube and subjected to a further 45 cycles of amplification using the same primers and reaction conditions. These products were used for restriction enzyme (RE) analysis with $M s p$ A1I and $C f o \mathrm{I}$ (Promega, Madison, WI). Following digestion the fragments, together with undigested product, were run in ethidium bromide $2.5 \%$ Metaphor gel (FMC Bioproducts, Rockland, ME, USA) in TAE buffer and bands were visualized under UV light. Genotypes were arbitrarily assigned based upon the similarity of the RE fragment patterns.

In addition to the conjunctival samples from the Kimberley outbreak, genotyping was also performed on routine genital samples from the same region 


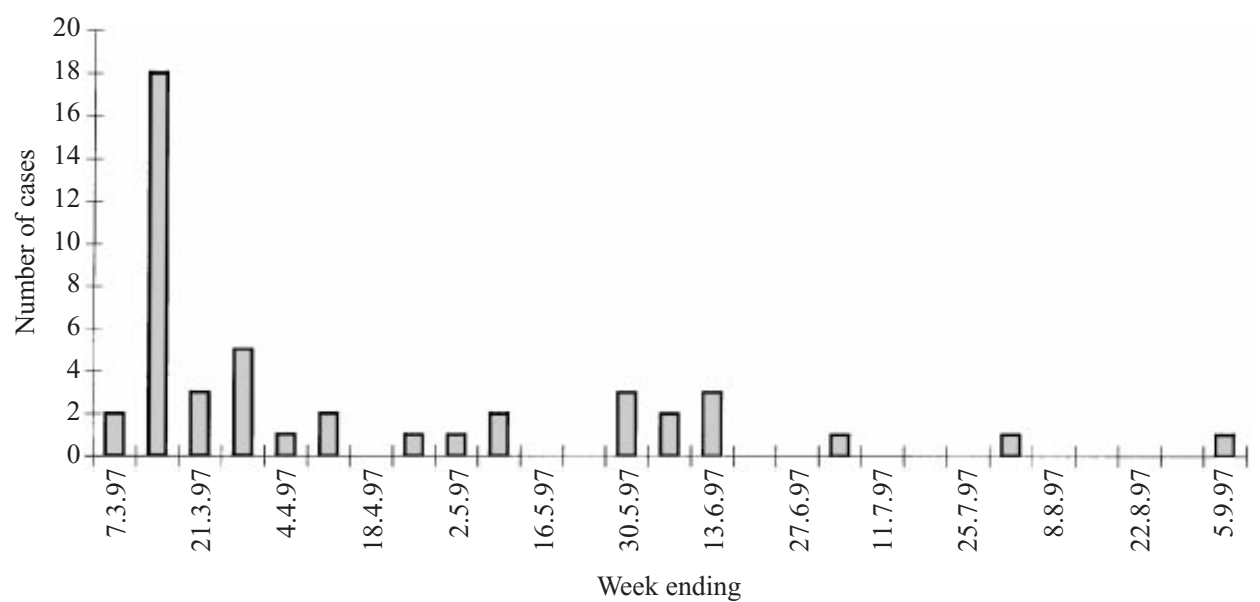

Fig. 1. Epidemic curve for the outbreak of gonococcal conjunctivitis occurring in the Kimberley region of Western Australia. Only laboratory-confirmed cases are included.

collected over the period of the outbreak. The Department of Microbiology at Royal Perth Hospital kindly supplied a number of genital isolates of $N$. gonorrhoeae from the Kimberley region of Western Australia. Samples from three cases of sporadic gonococcal conjunctivitis occurring during this period were also genotyped.

Several isolates from Central Australia were kindly supplied by The National Neisseria Reference Laboratory, Prince of Wales Hospital, Randwick, NSW. These included conjunctival isolates, a single isolate from a joint, and a small number of genital isolates collected during their epidemic of conjunctivitis. These isolates had been auxotyped and serotyped.

\section{RESULTS}

Forty-six cases involving 43 people occurred between March and September 1997 (Fig. 1). A 2 year-old boy had two separate infections 6 weeks apart and a 1 year-old boy had three separate infections. In each case, the second or third infections were several weeks after the previous one and followed a prolonged asymptomatic period. They were therefore included as new infections rather than relapses.

Forty of these patients lived in one of a cluster of four communities in the southeast Kimberley region. There is a high level of mobility between these communities as there are strong family and kinship associations between the residents. There is also frequent movement of people between these communities and Central Australian communities for the same reasons. The three other cases occurred in two other locations in southeast Kimberley, but all had been in contact with cases and/or contacts from the cluster of affected communities. The outbreak followed several months of unusually heavy rainfall in the area.

Three cases were in adults (aged 36, 43 and 60 years), 20 in children aged $0-4$ years, 15 in 5-9 yearolds and 7 in 10-14 year-olds. Twenty-eight cases were male and 15 were female. Attack rates were calculated for the 40 cases occurring in the cluster of four communities and were inversely proportional to age, being $425 \cdot 5 / 1000$ population in $0-4$ year-olds, $223 \cdot 8 / 1000$ in $5-9$ year-olds, $129 \cdot 6 / 1000$ in $10-14$ year-olds and 10.4/1000 in those aged 15 years and over.

Of the total 46 laboratory-confirmed infections, 37 were PCR positive only, 8 were culture and PCR positive, and 1 case was culture positive and PCR negative. Of the 45 PCR positive specimens, 24 could be genotyped and all showed identical restriction enzyme (RE) patterns (Fig. 2), designated genotype A. The remaining samples had either been discarded or failed to produce detectable amounts of DNA in the typing PCR.

We also tested 124 PCR-positive or culture-positive genital tract samples from patients in the Kimberley region during the same time period (Fig. 3). Of these, 24 failed to produce any detectable restriction enzyme patterns. Fifty-two had a consistent pattern called genotype B, while a further 34 had the same pattern but with a single additional band, which we designated genotype B1 (Fig. 2). The additional band was faint and was generally found in the product from the culture rather from the primary swabs. Fourteen other isolates showed a variety of RE patterns, none of which was genotype A. 


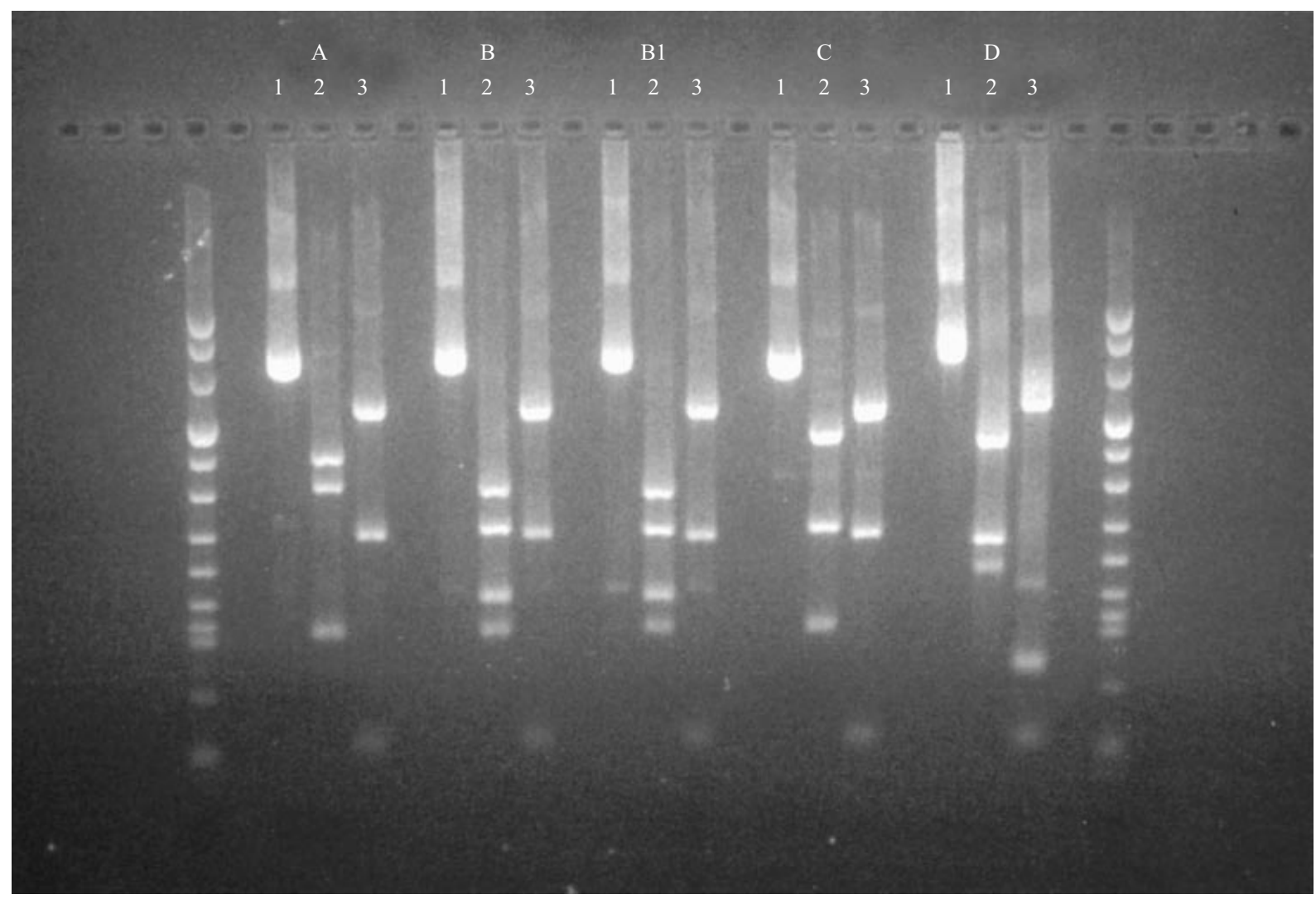

Fig. 2. Agarose gel stained with ethidium bromide showing the restriction enzyme patterns for Neisseria gonorrhoeae genotypes. The outer lanes contain Boeringher Mannheim molecular weight marker VIII, ranging from 1114 to 37 base pairs. Genotypes A, B, B1, C and D are illustrated. For each genotype, lane 1 is uncut PCR product, lane 2 is the product after being cut by MspA1I and lane 3 is product after being cut by $C f o \mathrm{I}$. The B1 genotype has a faint additional low molecular weight band.

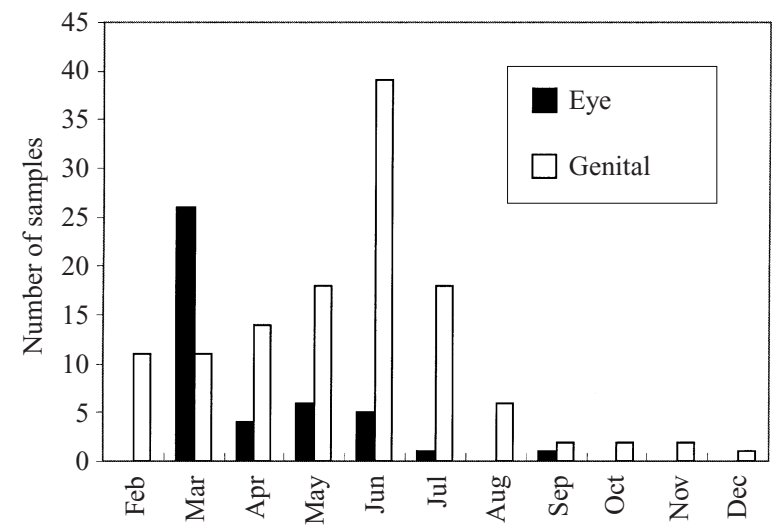

Fig. 3. Collection dates for epidemic conjunctival and genital tract samples from the Kimberley containing gonococci that were genotyped.

Three cases of sporadic gonococcal conjunctivitis were documented in northern Western Australia during the same period. Two occurred in the Kimberley region during August, in a neonate and a 3-year-old child from different communities. A further single case occurred in a neonate from the Pilbara
Table 1. Gonococcal genotypes detected in epidemic conjunctival and genital tract samples from the Kimberley region of Western Australia and from central Australia

\begin{tabular}{|c|c|c|c|c|}
\hline \multirow[b]{3}{*}{ Genotype } & \multicolumn{4}{|c|}{ Number of positive samples } \\
\hline & \multicolumn{2}{|c|}{ Epidemic conjunctival } & \multicolumn{2}{|l|}{ Genital } \\
\hline & Kimberley & $\begin{array}{l}\text { Central } \\
\text { Australia }\end{array}$ & Kimberley & $\begin{array}{l}\text { Central } \\
\text { Australia }\end{array}$ \\
\hline A & 24 & 9 & 0 & 0 \\
\hline $\mathrm{B} / \mathrm{B} 1$ & 0 & 0 & 86 & 2 \\
\hline Other & 0 & 0 & 14 & 0 \\
\hline
\end{tabular}

region (south of the Kimberley) in September. All three cases were due to genotype $\mathrm{B}$.

Twelve isolates from samples collected in Central Australia during March 1997 were also genotyped. The nine eye isolates and the single joint fluid isolate were all genotype A, whereas the two genital isolates were both genotype B (Table 1). All 12 had been 
shown to be Wt/IB3 at the National Neisseria Reference Laboratory (data not shown).

Cases were treated initially with procaine penicillin and probenecid, and contacts of cases with amoxycillin and probenecid. After it was found that some of the isolates were relatively resistant to penicillin, treatment was changed to ceftriaxone for index cases, and amoxycillin and probenecid for contacts. Due to the high incidence of co-infection with Chlamydia trachomatis (20/43 cases had this organism detected by PCR) many cases, and all contacts, also received azithromycin.

\section{DISCUSSION}

A widespread outbreak of gonococcal conjunctivitis involving the Kimberley region of Western Australia and Central Australia occurred during 1997. This appears to be the first recorded outbreak of conjunctivitis due to this organism reported or recorded in the Kimberley region of Western Australia. Since 1968, health care workers in this region have been encouraged to seek out cases of conjunctivitis and to collect gonococcal swabs from all cases of severe conjunctivitis. Therefore it is unlikely that any significant outbreak of gonococcal conjunctivitis in the previous 30 years would have been missed.

Previous evidence from limited auxotyping and serotyping had suggested that this outbreak was due to a single type which was also found to be causing some genital tract infections [4]. Using genotyping we were able to show that the gonococci responsible for genital and epidemic conjunctival infections were different, even if they shared the same auxotype/ serotype. This ability to augment conventional typing has been described previously [7] using genital isolates of $N$. gonorrhoeae. This study shows that it can also be used effectively for conjunctival samples, and that it can be used directly on patient samples. It is therefore especially valuable for situations where isolates are not available. This is a particular problem in remote area populations where the organism may not survive transport to the nearest laboratory.

All the epidemic conjunctival isolates were of a single genotype, which was the same in the Kimberley region and Central Australia. The temporal pattern of the outbreak [4] suggested that it began in the Kimberley and subsequently spread hundreds of kilometres to the east and south across the arid central regions. High rainfall, higher than average temperatures, high fly numbers and poor hygiene standards have been associated with outbreaks in Australia [3, 4], and in Africa [6]. Though flies have been implicated in person-to-person spread [11], it is unlikely that they could account for the rapid spread across the Kimberley and Central Australia. Over recent years there has been increased mobility of Aboriginal people between desert regions of Western Australia and Central Australia due to better vehicular access and increased attendance at ceremonial law and family activities. The spread of this outbreak follows this pattern of movement, and it seems most likely that infected individuals may have carried the organism to new areas. Early identification and treatment of infected individuals could prevent them carrying infection into unaffected populations.

Sporadic conjunctival and genital isolates collected across the period of the outbreak were all genetically distinct from the epidemic conjunctival ones. The three sporadic conjunctivitis cases were caused by strains of genotype B and $86 \%$ of the genital gonococci were either genotype $\mathrm{B}$ or the closely related genotype B1 (Fig. 2). Since the additional band in genotype B1 was faint, its absence from the genotype B samples may have been due to lower amounts of DNA that could not be detected on the gels, and the B and B1 patterns may still represent a single genotype. We did not find any evidence that the type causing the epidemic of gonococcal conjunctivitis originated from a genital reservoir, an observation that is consistent with the lack of association between conjunctivitis epidemics and genital gonorrhoea activity $[3,4,6]$.

Our population has an active programme for screening of individuals at risk of gonorrhoea, with over 5000 tests for genital tract infection being performed in the Kimberley population over the period of the conjunctivitis epidemic. This includes both symptomatic and asymptomatic individuals; therefore it is unlikely that we would have missed a significant genital reservoir of genotype A $N$. gonorrhoeae. However, as we were only able to genotype 100 gonococci from the Kimberley region, it is still possible that genotype A may be a very infrequent cause of genital infection and that even a low level may be sufficient to act as a source of epidemic conjunctivitis. Oropharyngeal infection is an alternative potential reservoir, but $N$. gonorrhoeae was detected in the oropharynx of only a small proportion of cases in a Central Australian outbreak [3]. Clearly, the origin of the gonococcal types causing epidemic conjunctivitis is not yet proven, but a genital reservoir 
is unlikely and public health interventions directed at control of genital infection in a community may not prevent conjunctivitis outbreaks.

Our experience with sporadic, rather than epidemic, cases of gonococcal conjunctivitis that occurred during this period was different, with the genotyping supporting a genital origin for the infection.

Epidemic gonococcal conjunctivitis is an ongoing problem in indigenous populations. Active surveillance and early laboratory confirmation is important in identifying and controlling outbreaks. Detection of gonococcal DNA using PCR is a valuable tool for the detection of this organism, and genotyping of PCRamplified DNA is an important tool in characterising outbreaks. Ideally it should be used in combination with conventional serotyping and auxotyping methods, but it may be the only method available in outbreaks such as this where there are few isolates. The reservoir for gonococci causing epidemic conjunctivitis remains unidentified, but does not appear to be the genital tract. Further work is needed to identify the reservoir if such epidemics are to be controlled. Until then, the emphasis must remain on early identification of cases and outbreaks, early treatment of cases and contacts, improvements in personal hygiene and possibly, restriction of movement of untreated cases and contacts between affected and unaffected communities.

\section{ACKNOWLEDGEMENTS}

We thank Dr John Tapsall of the National Neisseria Reference Laboratory for providing gonococcal isolates, and Ms Glenys Chidlow for assistance with the genotyping. We also thank Mr Tim Steele and Ms Karina Chaves for providing local census data for the communities, and the many health staff who assisted in the management of this outbreak.

\section{REFERENCES}

1 Brennan R, Patel M, Hope A. Gonococcal conjunctivitis in Central Australia. Med J Aust 1989; 150 : 48-9.

2 Monger K, Brennan R. Gonococcal conjunctivitis outbreak in a Northern Territory Aboriginal community. Commun Dis Intell 1992; 16: 534.

3 Merianos A, Condon R, Tapsall JW, et al. Epidemic gonococcal conjunctivitis in central Australia. Med J Aust 1995; 162: 178-81.

4 Matters R, Wong I, Mak DB. An outbreak of nonsexually transmitted gonococcal conjunctivitis in Central Australia and the Kimberley region. Commun Dis Intell 1998; 22: 52-8.

5 Thomson J, Lin M, Halliday L, et al. Australia's notifiable disease status, 1998. Commun Dis Intell 1999; 23: 277-308.

6 Mikru SM, Molla T, Ersumo M, et al. Communitywide outbreak of Neisseria gonorrhoeae conjunctivitis in Konso District, North Omo Administrative Region. Ethiop Med J 1991; 29: 27-35.

7 Lau QC, Chow VTK, Poh CL. Differentiation of Neisseria gonorrhoeae strains by polymerase chain reaction and restriction fragment length polymorphism of outer memberane protein IB genes. Genitourin Med 1995; 71 : 363-6.

8 Di Luca D, Mirandola P, Ravaioloi R, et al. Human herpesvirus 6 and 7 in salivary glands and shedding in saliva of healthy and human immunodeficiency virus positive individuals. J Med Virol 1995; 45: 462-8.

9 Hagblom P, Korch C, Jonsson A-B, Normark S. Intragenic variation by site-specific recombination in the cryptic plasmid of Neisseria gonorrhoeae. J Bacteriol 1986; 167: 231-7.

10 Miyada CG, Born TL. A DNA sequence for the discrimination of Neisseria gonorrhoeae from other neisseria species. Mol Cell Probes 1991; 5: 327-35.

11 Weinstein P. The Australian bushfly as a vector of Neiserria gonorrhoeae conjunctivitis. Med J Aust 1991; 155: 717. 